\title{
Main resonances in directly modulated semiconductor lasers: effect of spontaneous emission and gain saturation
}

\author{
C.Mayol, S.I.Turovets, R.Toral, C.R.Mirasso and L.Pesquera
}

\begin{abstract}
Boundaries for the primary saddle-node bifurcations related to the main resonances in pump-modulated laser diodes are obtained via numerical simulation. The model explicitly contains the gain saturation and spontaneous emission terms and the authors focus on the effect that these terms have on the large signal modulation regime. It is found that the spontaneous emission term strongly modifies the qualitative behaviour of the instability boundaries, while the gain saturation factor manifests itself in the damping of relaxation oscillations and leads to a simple quantitative shift of boundaries.
\end{abstract}

\section{Introduction}

High-speed modulation of laser diodes is of interest for developing large capacity information transmission and ultrafast optical processing systems [1-4]. Recently, a great deal of interest has been generated by the potential use of lasers running in a chaotic regime as the carriers of information in secure chaotic communication schemes [5-7]. In addition to the optical feedback and saturable absorption effects, chaos in laser diodes induced by pump current modulation or gain switching is another option for building transmitters for encoded optical communications. Although there has been some controversy in earlier theoretical [8-10] and experimental [1] results, it is now widely accepted that a single-mode laser diode with relatively small gain saturation and spontaneous emission factors might undergo a period doubling route to chaos under current modulation.

We have undertaken numerical calculations in the framework of the single mode rate equation model with the aim of determining the parameter domains of the basic instabilities involved. The influence of gain saturation factor [9] and of the spontaneous emission terms [10] have already been considered, but little attention has been given to main resonances. We understand the main resonance to be the maximum response of the system to external perturbation, quantified by the maximum intensity in the optical-power peak. Although main resonances have been considered for conventional class $B$ lasers theoretically and numerically

(C) IEE, 2001

IEE Proceedings online no. 20010245

DOI: 10.1049/ip-opt:20010245

Paper first received 26th June and in revised form 21st December 2000

C. Mayol, R. Toral and C.R. Mirasso are with the Departament de Física, Universitat de les Illes Balears, E-07071 Palma de Mallorca, Spain

C. Mayol and R. Toral are also with the Instituto Mediterráneo de Estudios Avanzados (IMEDEA, UIB-CSIC), E-07071 Palma de Mallorca, Spain

S.I. Turovets and L. Pesquera are with the Instituto de Física de Cantabria (CSIC) - Universidad de Cantrabria, Facultad de Ciencias, Avda. Los Castros s/n, E 39005 Santander, Spain

S.I. Turovets is now with Siros Technologies, Inc., 101 Daggett Drive, San Jose, CA 95134, USA
$[8,13-16]$ and also experimentally [17-19], the impact of large gain saturation and spontaneous emission terms is not yet fully understood and the aim of our work is to give more insight within this context.

\section{Model and results}

The dynamics of a single-mode semiconductor laser, a typical class $B$ laser, can be described in terms of two evolution equations, one for the slowly varying complex amplitude of the electric field inside the laser cavity $E$ and the other for the carrier number $N$ (or number of electronhole pairs). We consider the electric field $E$ normalised in such a way that its modulus square $I=|E|^{2}$ is equal to the number of photons inside the cavity. The equation for the electric field can be written in terms of this optical intensity $I$ and the phase $\phi$ by defining $E=\sqrt{ }(I) e^{i \phi}$. For simplicity, we neglect the explicit random fluctuations terms of these equations and retain, as usual [20], the mean power of the spontaneous emission. As the evolution equations for $I$ and $N$ do not depend on the phase $\phi$, we can concentrate only on the evolution of the former variables. The equations read

$$
\begin{aligned}
& \frac{d I}{d t}=[G(N, I)-\gamma] I+4 \beta N \\
& \frac{d N}{d t}=C-\gamma_{N} N-G(N, I) I
\end{aligned}
$$

where $G(N, I)$ is the material gain given by

$$
G(N, I)=g_{N} \frac{\left(N-N_{o}\right)}{1+\epsilon I}
$$

While the first term of the right-hand side of eqn. 1 accounts for the stimulated emission and losses, the last term, $4 \beta N$, accounts for the mean value of the spontaneous emission in the lasing mode. Eqns. 1 and 2 are written in the reference frame in which the frequency is zero at threshold when spontaneous emission noise is neglected. In these equations $C$ accounts for the number of carriers injected per unit time, $\gamma$ is the cavity decay rate, $\gamma_{N}$ is the carrier decay rate, $N_{o}$ is the number of carriers at transparency, $g_{N}$ is the differential gain parameter, $\epsilon$ the gain 
saturation parameter and $\beta$ is the spontaneous emission rate. Typical values of these parameters are: $C>$ threshold, $\gamma=0.5 \mathrm{ps}^{-1}, \quad \gamma_{N}=1 \mathrm{~ns}^{-1}, \quad N_{o}=1.5 \times 10^{8}, \quad g_{N}=1.5$ $\times 10^{-8} \mathrm{ps}^{-1}, \epsilon=10^{-8}-10^{-7}$ and $\beta \approx 10^{-8} \mathrm{ps}^{-1}$. The threshold value for $C\left(C_{t h}\right)$ is defined as the minimum number of carriers injected per unit time needed to fully compensate the losses and it is given by $C_{t h}=\gamma_{N} N_{t h}$ (neglecting spontaneous emission), where $N_{t h}=N_{o}+\gamma /$ $g_{N}$. The dynamics of these equations for constant $C>C_{t h}$ is such that both $I$ and $N$ relax to their steady states by performing damped oscillations $[20,21]$.

The purpose of this work is to study the dynamics of eqns. 1 and 2 when $C=C(t)$, i.e. it becomes a timedependent and controllable function, modelling the highspeed modulation of the laser at a given frequency $w_{m}$. More explicitly, we consider $C(t)=C_{b}\left[1+C_{m} \sin \left(w_{m} t\right)\right]$, where $C_{b}$ is a fixed value of the current such that $C_{b}>C_{t h}$. In our simulations we choose $C_{b}=1.2 C_{t h}$ and values for the relative amplitude of the modulation $C_{m}<1$, such that $C(t)>0$ for all times. When $C$ becomes time dependent, the dynamics are more complex than in the case of constant $C$, and a very rich dynamical structure can appear depending on the values of $C_{m}$ and $w_{m}$.

For small values of $C_{m}$ the system behaves almost as a linear oscillator with damping terms; the system oscillates periodically with the same frequency $w_{m}$ of the input current. To characterise the response of the system wo look at the maximum value of $I\left(I_{\max }\right)$ when we modulate the laser. It is well known that, under small signal modulation, the modulation response has a maximum at the relaxation oscillation frequency $[20,22]$ (resonance phenomenon). In what follows we take $w_{m}=m w_{o}$, being $w_{o}$ the relaxation oscillation frequency of the system for $C=C_{b}$ and $m$ a real number. The value of $w_{o}$ is, for the simplest case $\epsilon=\beta=0, w_{o} \approx \sqrt{ }\left(g_{N}\left(C_{b}-C_{t h}\right)\right)=23.5 \mathrm{~ns}^{-1}$ for our parameter values.
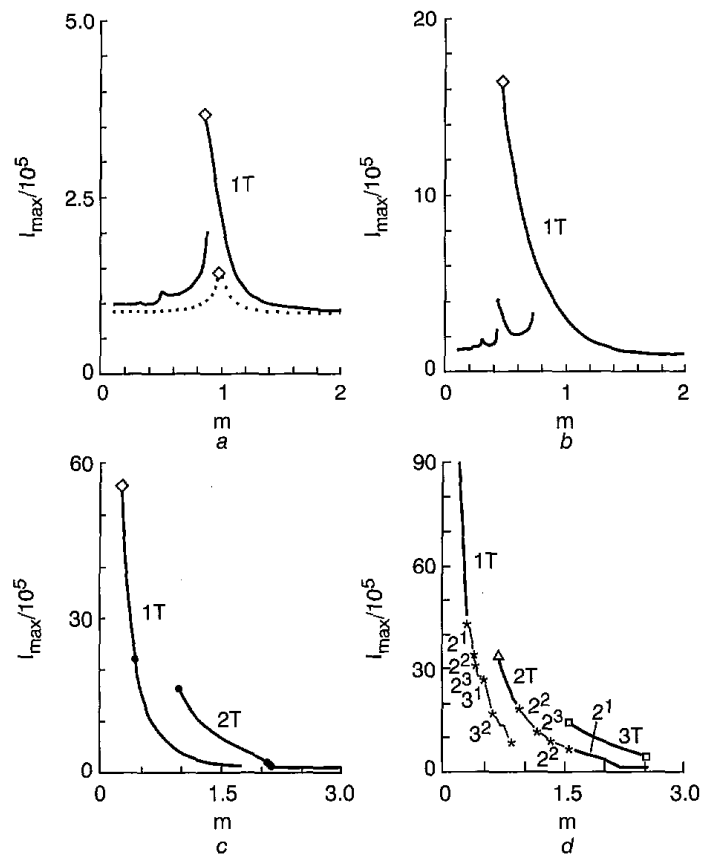

Fig. 1 Responses, $I_{m a x}$, against the normalised external frequency $m=w_{m} / w_{o}$ in the case $C_{b}=1.2 C_{t h}, \epsilon=0, \beta=0$ $a$ dotted line: $C_{m}=0.01$, solid line: $C_{m}=0.033$

$b C_{m}=0.1$

$c C_{m}^{m}=0.2$

$d C_{m}=0.3$

The $2^{k}$ responses correspond to further period doubling bifurcations

42
In contrast to the small signal response just described, large amplitude modulation, i.e. large values of $C_{m}$, give rise to strong nonlinear behaviours, since the nonlinear terms become relevant in the dynamics of the system. The maximum response (maximum value of $I_{\max }$ ) is not located any more at the relaxation oscillation frequency $w_{0}$ but is shifted to a smaller frequency. This fact can be seen in Fig. 1 where the response of the system, for different values of $C_{m}$, is shown. In this case we have taken $\epsilon=0$ and $\beta=0$ in eqns. $1-3$. Additionally, in this nonlinear regime, the response of the system to the external modulation can be such that other frequencies are excited besides the main frequency $w_{m}$. This fact gives rise to a more complex diagram for the response of the system, even allowing the phenomenon of multistability. The different possible responses of the system can be classified as $n / n_{1}$ where $n$ and $n_{1}$ are integer numbers with no common factors, such that the response frequency $w$ also bears the same relationship with respect to the modulation frequency $w_{m}$, i.e. $w=w_{m} n_{1} / n[22]$.

We will be mainly interested in primary resonances of the type $n / 1$. Our interest in these responses is based on the fact that they usually yield the maximum output power. These $n / 1$ resonances are also called $n T$ responses because the period of the resulting signal is $n$ times $T$, where $T=2 \pi / w_{m}$. Different types of stable $n T$ responses are shown in Fig. 1 for different values of the amplitude of the modulation, $C_{m}$, while in Fig. 2, the time evolution for

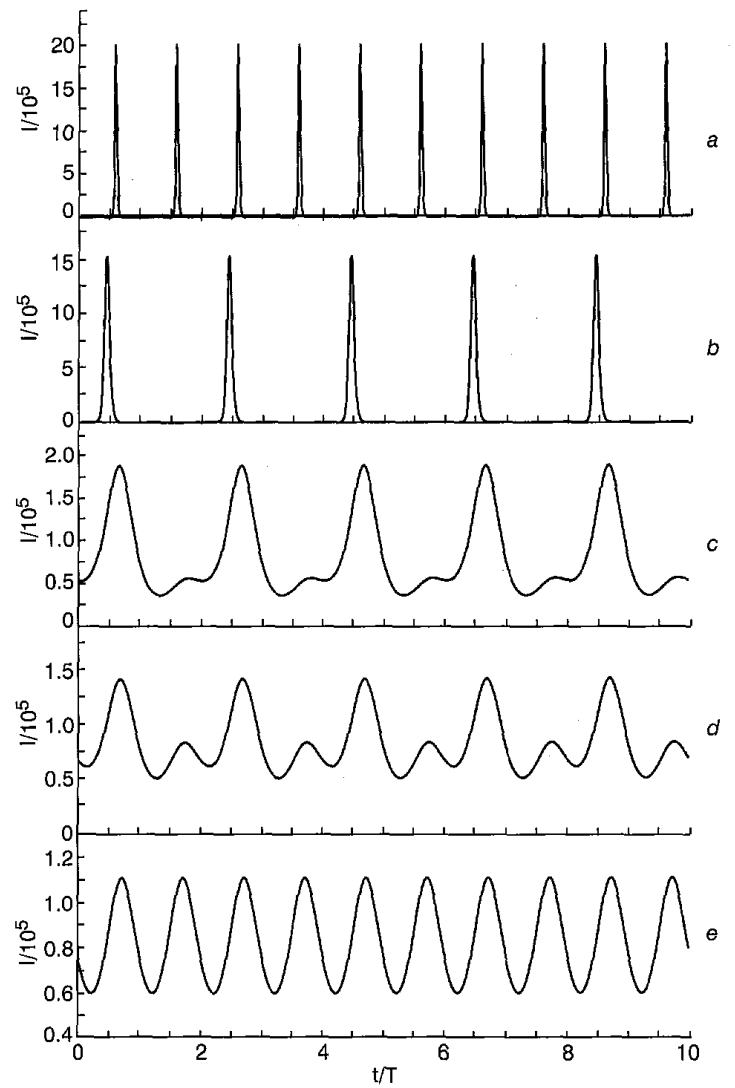

Fig. 2 Intensity against time for different values of $m$ for $C_{m}=0.2$ corresponding to the points marked as dots in Fig. $1 \mathrm{c}$

(a) $m=0.43$

(b) $m=0.97$

(c) $m=2.08$

(c) $m=2.08$

(d) $m=2.11$
(e) $m=2.12$

Same parameters as in Fig. 
the intensity is plotted for different values of the frequency $w_{m}$ and the common value $C_{m}=0.2$.

The qualitative picture described above can now be more explicitly detailed. For small modulation amplitude, see Fig. $1 a\left(C_{m}=0.01\right.$, dotted line $)$, the linear approximation applies and there are only single main resonances whose maximum lies approximately at the relaxation frequency. For larger modulation amplitudes, Fig. $1 a\left(C_{m}=0.033\right.$, solid line), the stable $1 T$ resonances destabilise themselves, via a saddle-node type instability, for sufficiently small values of the external frequency $w_{m}$ giving rise to a series of responses which are stable for some frequency ranges. This saddle-node instability is interesting since we have observed that the maximum response appears just before the solution becomes unstable. (We will come back to this point later). The same behaviour can also be observed in Fig. $1 b$ for a large modulation amplitude, $C_{m}=0.1$. When multistability is possible, and for those moderate levels of the amplitude of the modulation, the larger output intensity $I_{\max }$ always corresponds to the $1 T$ response. For larger values of $C_{m}$ there appear $n T$ responses with $n>1$. When these responses exist, their related output intensity exceeds that of the $1 T$ response. For example, in Fig. $1 c$ (corresponding to $C_{m}=0.2$ ), we see that the $1 T$ response, solid line, gives a smaller output than the $2 T$ response for the whole frequency range of existence of the $2 T$ response, namely for $m \in[0.968,2.118]$. Moreover, notice that the $1 T$ response does not exist (is unstable) in the frequency interval [1.761, 2.118].

For even larger values of $C_{m}$, the phase diagram of stable solutions becomes more complex, see Fig. 1d (corresponding to $C_{m}=0.3$ ). Besides obtaining $3 T$ resonances as the ones giving the maximum output intensity for some ranges of parameters, we can find period doubling bifurcations, period four etc., or even chaos, following the Feigenbaum route to chaos [22] or the route period doubling followed by period four, eight, period tripling, ..., as in $[9,10]$. These are indicated by thin lines and are denoted as $2^{k}$ responses, with being $k$ an indication of the number of period doubling bifurcations that the orbit has suffered. The same meaning applies for the $3^{k}$ responses.

In Fig. 2 we plot, for a fixed value of the modulation amplitude $\left(C_{m}=0.2\right.$, corresponding to Fig. $\left.1 c\right)$, time evolutions for the intensity $I(t)$ for several values of the frequency $m=w_{m} / w_{o}$. Case (a) corresponds to $m=0.43$ where, according to Fig. $1 c$, the $1 T$ type solution with maximum intensity appears. Case $(b)$ corresponds to a frequency $m=0.97$ where the $2 T$ solution begins to exist. We note that at this frequency, the $2 T$ signal has a maximum spectral component in the first harmonic, allowing for a clean time trace of the optical intensity, with no additional peaks, as shown in Fig. $2 b$. As the frequency increases, Figs. $2 c$ and $2 d$, the $2 T$ response deteriorates in the sense that the maximum intensity decreases and extra peaks develop in the time series. Finally, for $m=2.12$ the $1 T$ response is recovered. However, and in accordance with Fig. 1c, the maximum intensity is now much smaller than that of the optimal $1 T$ response shown in Fig. $2 a$.

In what follows, our main effort will be directed to give a whole description of the main resonances in the $\left(w_{m}, C_{m}\right)$ plane, by defining the lines of maximum response for each main resonance of the $n T$ type. This description can be of interest to the experimentalists since it allows determination of the resonance frequency at which the maximum response is obtained for a given external amplitude of the injection current.

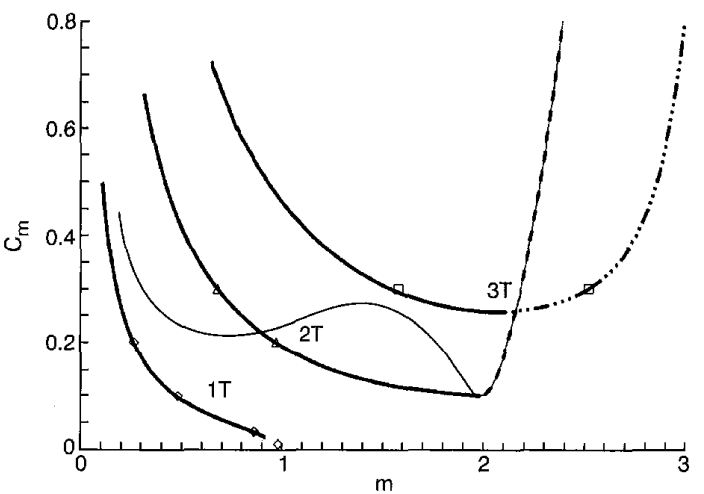

Fig. 3 Maxima of main resonances in the plane $\left(m, C_{m}\right)$ (solid thick lines)

Thin solid line: period doubling bifurcation of the $1 T$ resonance to $2^{1}$ (coincides with the limit of existence of resonance $1 T$ ), dashed line: lowest limit for the existence of the $2 T$ resonance, dot-dashed line: lowest limit for the existence of the $3 T$ resonance

With this aim in mind, we have performed intensive numerical simulations to obtain the maximum responses of the system for different $C_{m}$ and $w_{m}$. In principle, for a given $C_{m}$, one should find the value of $w_{m}$ that maximises the response at each $n T$ resonance. However, this is a very lengthy procedure that can be avoided by finding, instead, the value of $w_{m}$ where a saddle-node bifurcation is born, since, as we have already said, we have observed that the maximum response appears just before the solution becomes unstable. This allows us to identify the position of the maximum response in the $\left(w_{m}, C_{m}\right)$ plane with the position of the bifurcation. The procedure of finding such bifurcations is easier to implement using nonlinear dynamical tools than to perform whole simulations of the rate equations [23]. In Fig. 3 the primary saddle-node bifurcations are shown (thick solid lines) in the case $\varepsilon=0$ and $\beta=0$ for the $1 T, 2 T$ and $3 T$ resonances. We show in some cases that the location of the saddle-node bifurcations coincides, with great accuracy, with that of the maximum $I_{\max }$. The latter have been obtained by numerical simulations of the laser equations and are indicates by symbols in the Figure. Notice, however, that the line of the saddlenode bifurcations cannot reach the linear limit $m=1$ corresponding to very low amplitude of the modulation. We also plot in the same Figure some additional lines that correspond to domains of existence of the abovementioned $n T$ resonances. Within these domains, besides the 'pure' $n T$ solutions, there exist a rich variety of bifurcated solutions (period doubling, period 4 and so on, indicating the route to chaos described previously). The $1 T$ solution only exists below the thin solid line of Fig. 3 . The $2 T$ solution is limited by the thick solid line of $2 T$ and the dashed line and it only exists within this limit. Finally, the $3 T$ solution is limited by the thick solid line of $3 T$ and the dot-dashed line of the Figure. In what follows, we will restrict ourselves to the thick lines denoting the maximum of each resonance.

We now describe the effect of the gain saturation term. We plot in Fig. 4, the changes on the line of main resonances (given in Fig. 3 for $\epsilon=\beta=0$ ) in the case $\epsilon \neq 0$ (but still $\beta=0$ ). It can be seen clearly that the saturation term does not change qualitatively, neither does the location of the lines nor the overall dynamical behaviour of the system. The only difference is that, for a fixed frequency, a larger value of $C_{m}$ is needed to obtain the optimal periodic response. This is clearly compatible with 


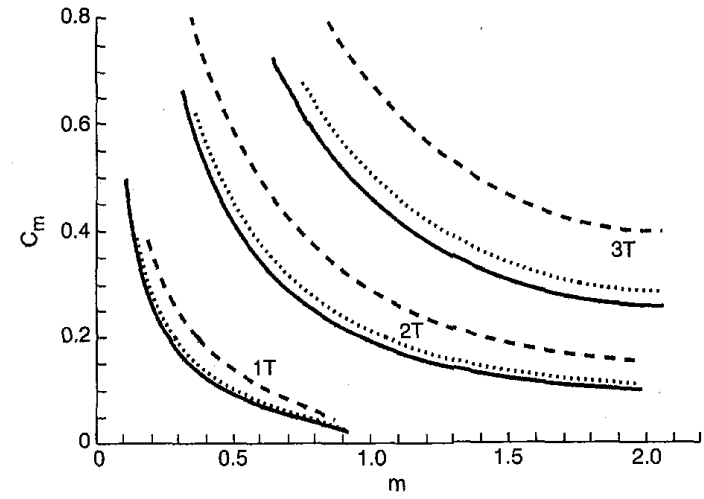

Fig. 4 Maxima of main resonances in the plane $\left(m, C_{m}\right)$ solid line: $\epsilon=0$, dotted line: $\epsilon=6 \times 10^{-9}$, dashed line: $\epsilon=3 \times 10^{-8}, \beta=0$ Other parameters as in Fig. 1

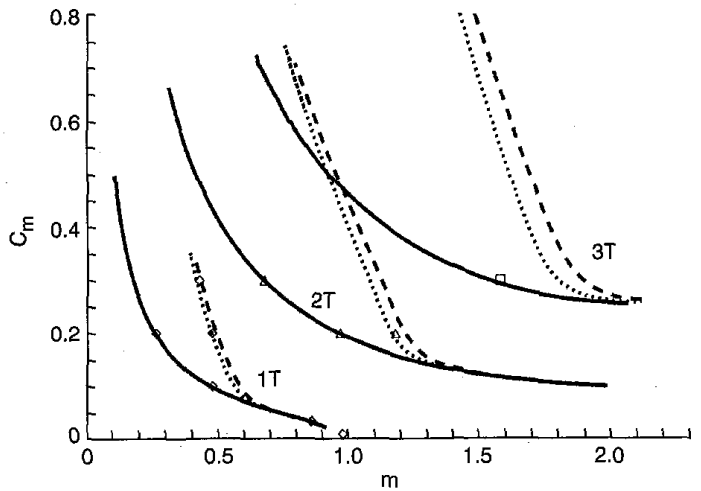

Fig. 5 Maxima of main resonances in the plane $\left(m, C_{m}\right)$ solid line: $\beta=0$, dotted line: $\beta=6.2 \times 10^{-11} \mathrm{ps}^{-1}, \beta=2.3 \times 10^{-10} \mathrm{ps}^{-1}, \epsilon=0$
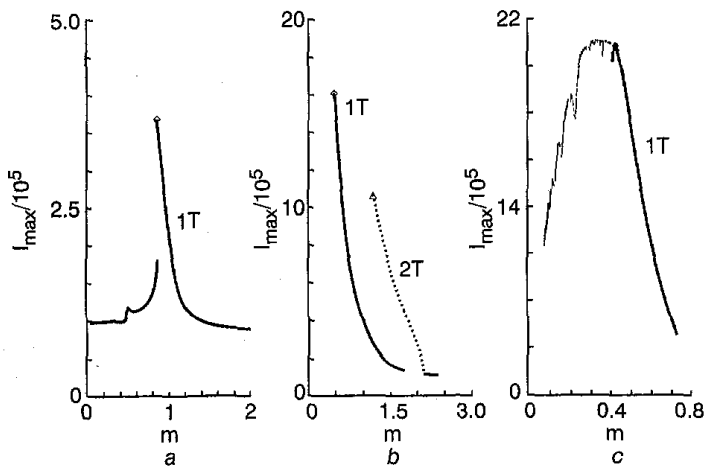

Fig. 6 Responses, $I_{m a x}$, against the normalised external frequency $m$ $C_{b}=1.2 C_{t h}, c=0, \beta=6.2 \times 10^{-11} \mathrm{ps}^{-1}$

$a C_{m}=0.033$

$b C_{m}=0.2$

c $C_{m}=0.3$

the fact that the main effect of the saturation term is to increase the dissipation.

On the contrary, the effect of the spontaneous emission term strongly changes the response of the system. In Fig. 5, - the lines of $I_{\max }$ in the $\left(w_{m}, C_{m}\right)$ plane are shown for the case $\beta \neq 0$ and $\epsilon=0$. One can observe the dramatic change in the behaviour of the response of the system for small frequencies with respect to that observed for $\beta=0$ (solid line). When increasing the modulation amplitude we find a steep response that indicates that the frequency $w_{m}$ for the optimal response $I_{\max }$ is less sensitive to the amplitude of the external modulation $C_{m}$. We speculate that this effect might be due to the fact that for $\beta \neq 0$ there is a background of photons preventing the intensity decreasing below a certain value, so yielding a frequency-independent response. For the case $\epsilon \neq 0$ and $\beta \neq 0$ we observe the same qualitative results as those shown in Figs. 4 and 5. It is important to point out that values of $\epsilon$ and $\beta$ different from zero yield chaos suppression, a fact that has been already pointed out both numerically and experimentally. Naively, one could expect that the gain saturation parameter plays a more important part in the dynamics. However, we observe that in some situations the main resonances can be affected more strongly by the spontaneous emission factor than by the gain saturation factor.

In the absence of spontaneous emission, $\beta=0$, the tendency to decrease the main resonance frequency with increasing amplitude of modulation can be explained using a Toda-like potential function $[21,24]$. Physically, it means that a maximum number of photons in the cavity is available for modulation. However, in the presence of spontaneous emission terms, this is not true anymore and the Toda potential as a function of the intensity changes, becoming more symmetric and steeper for very low intensities. This means that the spontaneous emission background is not available for modulation and it is maintained essentially unchanged. As soon as the intensity of the laser reaches this background level, the dynamics change and at low frequencies a nonresonant regime of gain switching (with no dependence on frequency in some modulation frequency intervals observed) dominates. In Fig. 6, we plot the maximum intensity responses $I_{\max }$ against frequency $w_{m}$ for different values of $C_{m}$, in the case $\beta \neq 0$. Compared with Fig. 1, we notice that at small modulation amplitude $\left(C_{m}=0.033\right.$, Fig. $\left.6 a\right)$ the response for small values of $w_{m}$ does not change qualitatively and very little quantitatively with respect to that of Fig. la. However, for large values of $C_{m}$, the effect of $\beta$ becomes more evident. At $C_{m}=0.2$ (Fig. 6b) the maximum response for the resonance $1 T$ is now at a large frequency than in Fig. $1 b$ and, moreover, the corresponding value of $I_{m a x}$ is much smaller. Increasing $C_{m}$ even further $\left(C_{m}=0.3\right.$ in Fig. $\left.6 c\right)$, we find that the maximum response for the resonance $1 T$ (thick line) is much smaller than in the case of Fig. $1 d$, and corresponds to a much larger frequency. It has to be mentioned that in this case, the value of $w_{m}$ that maximises the $1 T$ response presents larger differences in the location of the saddlenode than in previous cases. (This difference can be seen in Fig. $6 c$ as the difference between the maximum and the end of the solid line.) In Fig. $6 c$ we also plot other responses for the smallest values of $w_{m}$. These responses, while having a larger output than the $1 T$ responses, can either have a period larger than $1 T$ or, while still being $1 T$, have extra peaks in the time series. They include responses of period $1 T, 2 T, \ldots$, being an example of the structure also reported in [25]. We also point out that there exists a flat response for some ranges of values of $w_{m}$.

Our numerical results can be compared with the experiments. In particular, a $2 T$ resonance regime has been readily identified experimentally in different kinds of laser diodes $[1,19,26]$. This leads to sharp spikes like those presented in Fig. 2. Particularly relevant to our work is the paper by Liu and Ngai [1] where the response of a single-mode DFB laser subjected to current modulation is considered. They observe the following features when changing the modulation frequency and amplitude of the RF signal: 
(a) For small modulation frequency there is only a $1 T$ period response for any signal amplitude. This behaviour also appears in our system if we modulate with a normalised frequency $m<0.2$ (see Figs. 3 and 4).

(b) For intermediate modulation frequency there is a transition from $1 T$ to $2 T$ responses when increasing the modulation amplitude. In our case, this behaviour can be inferred from Fig. 1. However, a further transition from $2 T$ back to $1 T$ is experimentally, but not numerically, observed.

(c) $3 T$ and $4 T$ solutions appear for large enough modulation frequency and amplitude. We find these solutions also in the case of large enough values of the modulation frequency and amplitude. (The range of existence of the $3 T$ solution is plotted in Fig. 1d. while the $4 T$ solution would appear for larger values of the amplitude that are not plotted in the Figure.)

\section{Conclusions}

We have undertaken a numerical study to identify the optimal response of a class $B$ laser subjected to an external periodic modulation in the pump of relative amplitude $C_{m}$ and frequency $w_{m}$. We have computed the lines in the $\left(w_{m}, C_{m}\right)$ plane that gives a maximum response for each type of $n T$ resonance. The influence of saturation and spontaneous emission terms on the dynamics has also been examined. We have found that these specific laser diode parameters increase the damping of relaxation oscillations, thus increasing thresholds of instabilities in the system. Our results qualitatively agree in part with the experimental observations with $1.55 \mu \mathrm{m}$ InGaAs DFB lasers [1].

\section{Acknowledgments}

We acknowledge financial support from DGES (Spain) projects PB97-0141-C02-01, BMF 2000-1108 and TIC 99-0645. S.I. Turovets acknowledges the fellowship from MEC (Spain)

\section{References}

1 LIU, H.F., and NGAI, W.F.: 'Nonlinear dynamics of a directly modulated $1.55 \mathrm{um}$ InGaAsP distributed feedback semiconductor aser', IEEE J. Ouantum Electron 1995,29 (6), pp $1668-1675$

2 MIRASSO, C.R., COLET, P., and SAN MIGUEL, M.: 'Dependence of time jitter on bias level for single-mode semiconductor lasers under high speed modulation', IEEE J. Quantum Electron., 1993, 29, pp. 23-31
3 COLET, P., MIRASSO, C.R., and SAN MIGUEL, M.: 'Memory diagram of single-mode semiconductor lasers', IEEE $J$. Quantum Electron., 1993, 29, pp. 1624-1630

4 BREUER, D., and PETERMANN, K.: 'Comparison of NRZ- and RZmodulation format for $40-\mathrm{Gb} / \mathrm{s}$ TDM standard-fiber systems', IEEE Photonics Technol. Lett., 1997, 9, pp. 398-400

5 MIRASSO, C.R., COLET, P., and GARCÍA-FERNÁNDEZ, P.: 'Synchronization of chaotic semiconductor lasers: Application to encoded communications', IEEE Photonics Technol. Lett., 1996, 8, pp. 299-301

6 GOEDGEBUER, J.P., LARGER, L., and PORTE, H.: 'Optical cryptosystem based on synchronization of hyperchaos generated by a delayed feedback tunable laser diode', Phys. Rev. Lett., 1998,80 , pp. 2249-2252

7 FISHER, I., LIU, Y., and DAVIS, P.: 'Synchronization of chaotic semiconductor laser dynamics on sub-ns timescales and its potential for chaos communication', Phys Rev, A, 2000, 62, R011801

8 ERNEUX, T., BAER, S.M., and MANDEL, P.: 'Subharmonic bifurcation and bistability of periodic solutions in a periodically modulated laser', Phys. Rev. A, 1987, 35, pp. 1165-1171

9 LAMELA, H., CARPINTERO, G., and ACEBO, P.: 'Truncation of the Feigenbaum sequence in directly modulated semiconductor lasers', IEEE J. Quantum Electron., 1998, 34, (3), pp. 491--496

10 LAMELA, $H$, CARPINTERO, $G$, and MANCEBO, $\mathrm{F}$. 'Period tripling and chaos in the dynamic behavior of directly modulated diode lasers', IEEE J. Quantum Electron., 1998, 34, (10), pp. 1797-1801

11 AGRAWAL, G.P.: 'Effect of gain nonlinearities on period doubling and chaos in directly modulated semiconductor lasers', Appl. Phys. Lett., 1986, 49, (16), pp. 1013-1015

12 HORI, Y., SERIZAWA, $\mathrm{H}$, and SATO, H.: "Chaos in a directly modulated semiconductor laser', J. Opt. Soc. Am. B, 1988, 5, (5), pp. $1128-1132$

13 SAMSON, A.M., and TUROVETS, S.I.: 'Hierarchy of bifurcations in a laser with periodic modulation of losses', Dokl. AN BSSR, 1987, 31, pp. $888-890$

14 SCHWARTZ, I.B.: 'Infinite primary saddle-node bifurcation in periodically forced systems', Phys. Lett. A, 1988, 126, (7), pp. 411-418

15 SAMSON, AM., LOGVIN, Yu.A., and TUROVETS, S.I. 'The role of potential symmetry and shape of a nonlinear oscillator in the bifurcation potential symmetry and shape of a nonlinear oscillator in the bifurcation hierarchy for harmon
1990,33 , pp. $40-50$

16 SCHWARTZ, I.B., and ERNEUX, T.: 'Subharmonic hysteresis and period doubling bifurcations for a periodically driven laser', SIAM $J$ Appl. Math., 1994, 54, (4), pp. 1083-1100

17 SAMSON A M TUROVETS, S I CHIZHEVSKY VN and CHURAKOV, VV: 'Nonlinear dynamics of a loss-switched $\mathrm{CO}_{2}$ laser', Sov. Phys.-JETP, 1992, 74, (4), pp. 628-639

18 CHIZHEVSKY, V.N., and TUROVETS, S.I.: 'Small signal amplification and classical squeezing near period-doubling bifurcations in a modulated $\mathrm{CO}_{2}$-laser", Opt. Commun., 1993, 102, pp. 175-182

19 BENNETT, S., SNOWDEN, C.M., and IEZEKIEL, S.: 'Nonlinear dynamics in directly modulates multiple-quantum-well laser diodes' IEEE J. Quantum Electron. 1997, 33, (11), pp. 2076-2083

20 AGRAWAL, G.P., and DUTTA, N.K.: 'Long-wavelength semiconductor lasers' (Van Nostrand Reinhold, New York, 1986)

21 MAYOL, C., TORAL, R., and MIRASSO, C.R.: 'Lyapunov-potential description for laser dynamics', Phys. Rev. A, 1999, 59, pp. 4690-4698

22 JACKSON, E.A.: 'Perspectives of nonlinear dynamics' (Cambridge University Press, Cambridge, 1989)

23 DOEDEL, E, FAIRGRIEVE, T, SANDSTEBE, B, CHAMPNEYS, A. KUTZNETZOY, Yu, and WANG, S. 'AUTO97: Continuation and bifurcation software for ordinary differential equations'. and bifurcation software for ordinary

24 OPPO, G.L., and POLITI, A:: 'Toda potential in laser equations', $Z$ Phys. B, Condens Matter, 1985, 59, pp. 111-115

25 TANG, M., and WANG, S.: 'Simulation studies of bifurcation and chaos in semiconductor lasers', Appl. Phys Lett, 1986, 48, (14) pp 900-902

26 WADA, K., MARUI, H, HORINAKA, H, and CHO, Y: 'Disappearance of chaos on a $0.8 \mu \mathrm{m}$ AlGaAs Fabry-Perot semiconductor laser modulated near the relaxation oscillation frequency', Opt. Commun., 1998, 155, pp. 301-306 